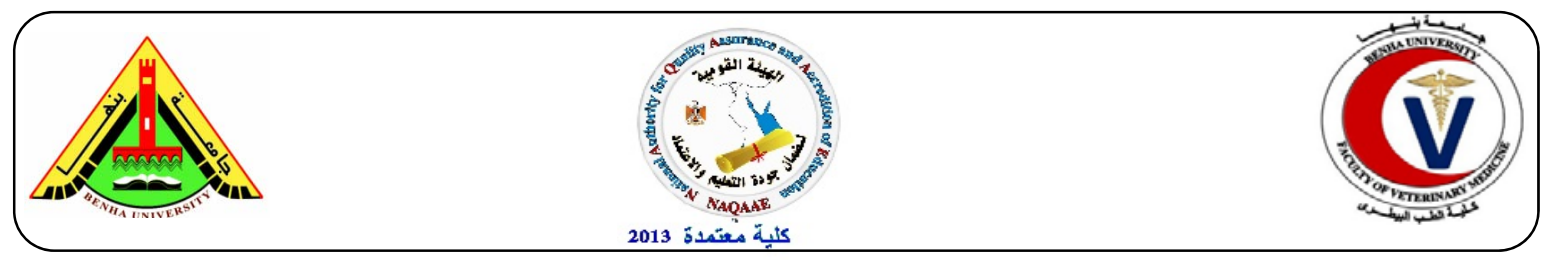

\title{
Rapid tests for detection of ciprofloxacin residues in liquid milk
}

\author{
Hamdi A. Mohammed ${ }^{1}$, Adham M. Abdou², Amal M. Eid², Asem M. Zakaria ${ }^{2}$ \\ ${ }^{1}$ Department of Food Control, Faculty of Veterinary Medicine, Benha University. ${ }^{2}$ Animal Health \\ Research Institute, Tanta Branch. ${ }^{3}$ Department of Food Control, Faculty of Veterinary Medicine, Aswan \\ University
}

\section{A B S T R A C T}

A total of 234 randomly selected milk samples from different sources (110, 50, 34 and 40 samples) from each of (market milk, collecting centers, bulk tank and UHT milk samples; respectively) were analyzed for the quinolones residues by rapid quinolones detection strip test. Results revealed that $17.3 \%, 14 \%$ and $20.6 \%$ of milk samples were positive for the presence of quinolones residues for market milk, collecting centers and bulk tank milk samples; respectively, while not detected in UHT milk samples. The positive milk samples were subjected to HPLC analysis for quantitative detection of ciprofloxacin residues, which were present in $11.8 \%, 8 \%$, and $8.8 \%$ for market milk, collecting centers and bulk tank milk samples; respectively, with mean values of $17.37 \pm 0.27,18.02 \pm 0.7$ and $17.79 \pm 0.75 \mathrm{ppb}$; respectively. None of them had a concentration exceeded the maximum residual limit set by different international standards. Public health importance of ciprofloxacin residues was discussed.

Keywords: Rapid Detection, Ciprofloxacin, Liquid Milk.

(http://www.bvmj.bu.edu.eg)

(BVMJ-30(1): 246-253, 2016)

\section{INTRODUCTION}

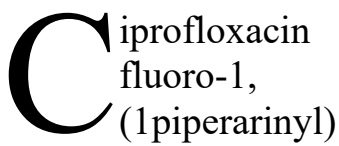

(1-cyclopropyl-64-dihydro-4-oxo-73-quinolone carboxylic acid) is a synthetic quinolone anti-infective agent. The drug is structurally related to other quinolones including cinoxacin, enoxacin, lomefloxacin, nalidixic acid, norfloxacin, ofloxacin and pefloxacin. Ciprofloxacin is a fluoroquinolone synthetic antimicrobial used in the treatment of severe and invasive infections in humans and animals. Ciprofloxacin is the cheapest fluoroquinolone and is often prescribed for the treatment of animals. Probably the most important threat to human health as a result of fluoroquinolone use in animal production is the development of fluoroquinoloneresistant bacteria as it has been discovered that a major route of transmission of resistant micro-organisms from animals to humans is through the food chain. The misuse of this drug is one of the causes of drug residues in milk (Adekunbi and Andrew, 2015). Milk with fluoroquinolones residues may lead to their accumulation in the cartilage of long bones and produce irreversible arthropathy with functional impairment in the fetus. FDA (Food and Drug Administration) includes ciprofloxacin in category $\mathrm{C}$ of risk in pregnancy (Filip et al., 2014). Low levels of ciprofloxacin can strongly suppress facultative anaerobic human intestinal microflora (Cerniglia and Kotarski 1999). Some fluoroquinolones have also been suspected to affect the central nervous systems. For example, in human, they have been associated in some cases of severe disorders like headache, dizziness or convulsions (Jinqing et al., 2010). Moreover, direct toxicity and conditions such as phototoxic skin reactions in humans can be induced by fluoroquinolones. 
Immunological tests cannot identify which antibiotic are present in the milk and may detect antibiotic residues at levels far below the officially mandated safe levels. Therefore, sensitive and specific analytical techniques for the identification and quantitation of antibiotic residues in milk are required. Liquid chromatography (LC) is a technique most commonly used for this purpose (Navratilova et al., 2011). The efficient control of fluoroquinolones residues in milk, especially ciprofloxacin, would require screening tests which give rapid results and allow simultaneous analysis of large numbers of samples. A post-screening step for positive screening samples must be confirmed, generally with chromatographic methods (quantitative method) (Sanz et al., 2015). Therefore, milk samples were examined for the presence of ciprofloxacin residues by screening rapid test then the results were quantitatively detected by using high performance liquid chromatography (HPLC) analysis, then the results were compared with the maximum residual limit set by different international standards.

\section{MATERIAL AND METHODS}

\subsection{Samples}

A total of 234 randomly selected milk samples obtained from different sources $110,50,34$ and 40 samples from each of market milk, collecting centers, bulk tank and UHT milk; respectively to estimate the presence of ciprofloxacin residues. The samples were collected individually $(500 \mathrm{ml}$ of each) in screw-capped bottles, then were transferred to laboratory in ice box within $1 \mathrm{hr}$ and stored at refrigerator till to be examined.

\subsection{Qualitative detection of quinolones residues in milk samples}

Rapid quinolones detection strip test (Nadezhda et al., 2014). Materials: Kit contain 100 quinolones rapid test strips, pipettes and quinolones diluent were purchased from Shenzhen Lvshiyuan Biotechnology Co., Ltd D Building,
National Biological Industrial Park of Marinelife, No.2 Binhai Road, Dapeng, Shenzhen, 518120, China. The test has been applied according to the manufacturer instructions. The test was based on immunechromatographic technology. Quinolones in sample in the process of flow were combined with the colloidal gold-labeled monoclonal antibody, inhibited the antibody combining with Quinolone conjugate binding present in the $\mathrm{T}$ line on the test strip. If the content of Quinolone in the sample is high, $\mathrm{T}$ line is lighter than $\mathrm{C}$ line or $\mathrm{T}$ line is invisible.

\subsection{Quantitative detection of ciprofloxacin residues in milk samples}

The milk samples have been examined for the presence of ciprofloxacin by a quantitative method as previously described by Verdon et al (2004). HPLC type: High performance liquid chromatography Agilent series 1050 quaternary gradient pump, Series 1050 auto sampler, series 1050 UV- Vis detector, and HPLC 2D chemstation software (Hewlett-Packard, Les Ulis, Germany). The chromatographic column was a reversed-phase column (Extend-c18, Zorbax column $4.6 \mathrm{~mm}$ i.d, $250 \mathrm{~mm}, 5 \mu \mathrm{m}, \quad$ Agilent Co). HPLC condition: Injection volume $20 \mu$, flow rate $1 \mathrm{ml} / \mathrm{min}$, column temperature $50^{\circ} \mathrm{C}$. UVdetector wave length: $280 \mathrm{~nm}$ and the mobile phase: acetic acid $(50 \mathrm{~mL} / \mathrm{L}$ distilled water): acetonitril: methanol (900: 50: 50). Samples preparation: $2 \mathrm{ml}$ of milk sample were added to $8 \mathrm{ml}$ of $5 \%$ Trichloro acetic acid (TCA) then mixing by Vortex-mix for $1 \mathrm{~min}$ after that we make rotary agitate for 10 mints followed by centrifugation for 5 mints $\left(14000 \mathrm{rpm}+4^{\circ} \mathrm{C}\right)$ then filtrate supernatant on $0.45 \mu \mathrm{m}$ nylon filter and inject $20 \mu \mathrm{l}$ in the LC instrument. (liquid/liquid extraction method) and stock standard solution of ciprofloxacin were prepared at concentration of $(50,100,250$, 500,1000 and 1500) ppb. The calibration curves were generated and quantification of residues in the sample was obtained and 
calculated from area under curves extrapolated automatically by the software.

\section{RESULTS}

Results in Table (1) showed that $17.3 \%$, $14 \%$ and $20.6 \%$ of milk samples were positive for the presence of quinolones residues for market milk, collecting centers and bulk tank milk samples; respectively, while not detected in UHT milk samples. The statistical analysis revealed that the difference between the percentages of quinolones residues presence in milk samples from different sources was significant $(\mathrm{P}=0.034)$.
Results in Table (2) showed that the percentages of presence of ciprofloxacin residues in market milk, collecting centers and bulk tank milk samples were 11.8, 8, and 8.8 with a mean value of $17.37 \pm 0.27$, $18.02 \pm 0.7$ and $17.79 \pm 0.75 \mathrm{ppb}$; respectively. The statistical analysis revealed that the difference between the mean values of ciprofloxacin residues in milk samples were none significant $(\mathrm{P}=$ 0.564). Results in Table (3) declared that none of positive milk sample had ciprofloxacin residue concentration exceeded the maximum residual limits compared to different international standards.

Table (1) Statistical analytical results of rapid quinolones detection strip test

\begin{tabular}{lccccc}
\hline Quinolones residues & Market milk & $\begin{array}{c}\text { Milk collecting } \\
\text { centers }\end{array}$ & Bulk tank & UHT milk & P - value \\
\hline Examined samples & 110 & 50 & 34 & 40 & \\
No of +ve & 19 & 7 & 7 & 0 & 0.034 \\
\% of +ve & 17.3 & 14 & 20.6 & 0 & 0.034 \\
\hline
\end{tabular}

Table (2) Descriptive statistical results of ciprofloxacin residues in examined milk samples measured by HPLC

\begin{tabular}{lcccc}
\hline $\begin{array}{l}\text { Ciprofloxacin } \\
\text { residues }\end{array}$ & Market milk & $\begin{array}{c}\text { Milk samples } \\
\text { Milk collecting } \\
\text { centers }\end{array}$ & Bulk tank & P - value \\
\hline Examined samples & 110 & 50 & 34 & \\
No of +ve & 13 & 4 & 3 & \\
$\%$ of + ve & 11.8 & 8 & 8.8 & \\
Min. & 16.28 & 16.34 & 16.59 & \\
Max. & 19.47 & 19.25 & 19.19 & 0.564 \\
Mean \pm SE* $^{*}$ & $17.37 \pm 0.27$ & $18.02 \pm 0.7$ & $17.79 \pm 0.75$ & \\
\hline
\end{tabular}

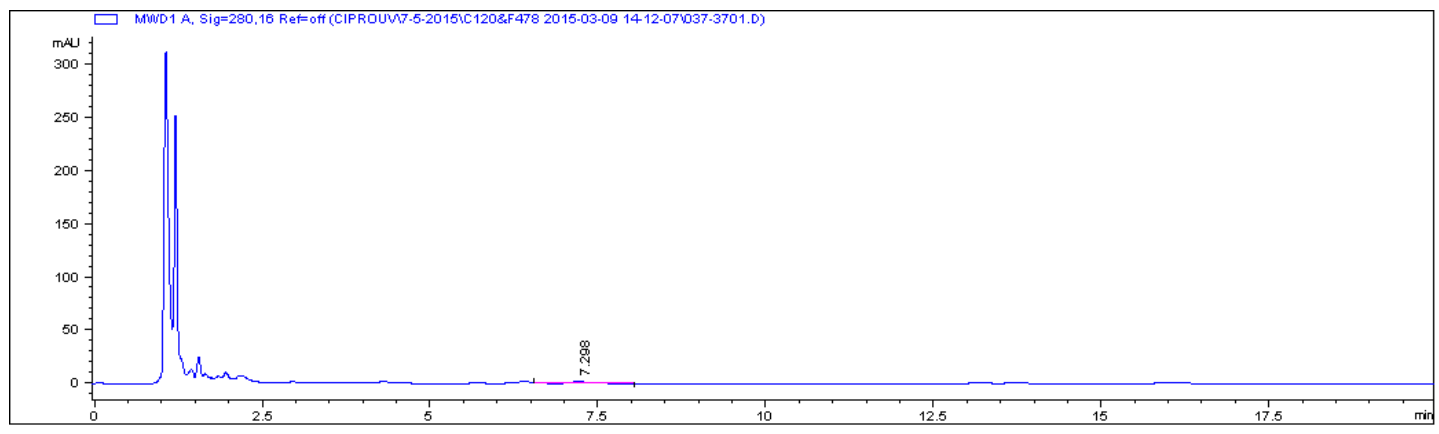

Minimum concentration of ciprofloxacin residues detected in market milk samples (16.28ppb) 


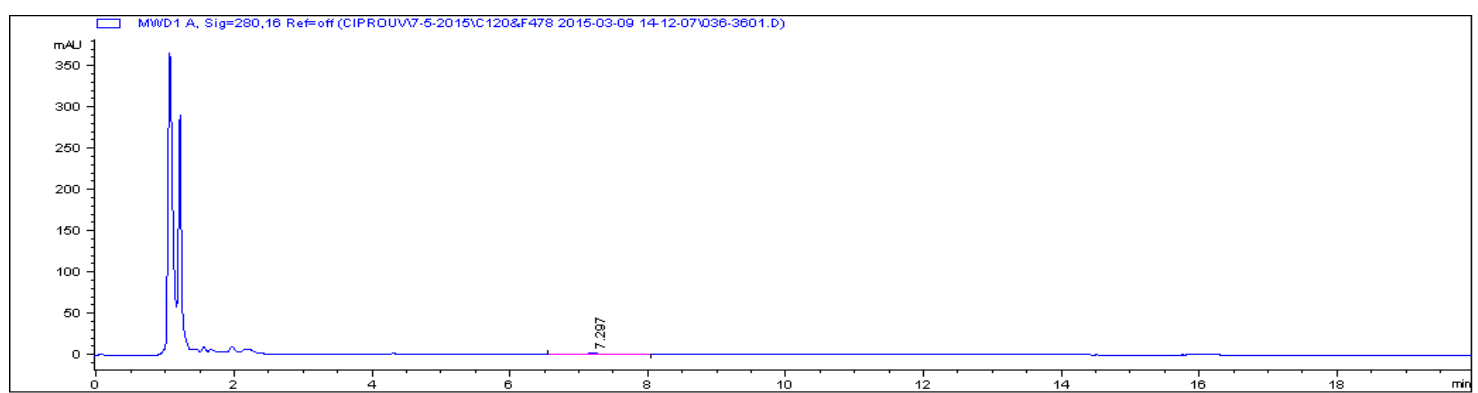

Maximum concentration of ciprofloxacin residues detected in market milk samples (19.47ppb).

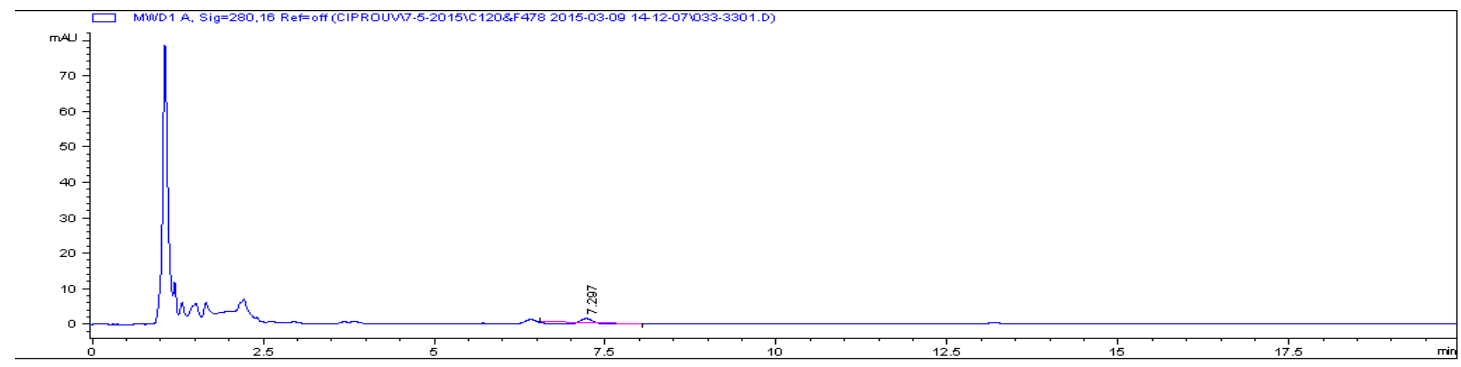

Minimum concentration of ciprofloxacin residues detected in collecting centers milk samples (16.34ppb).

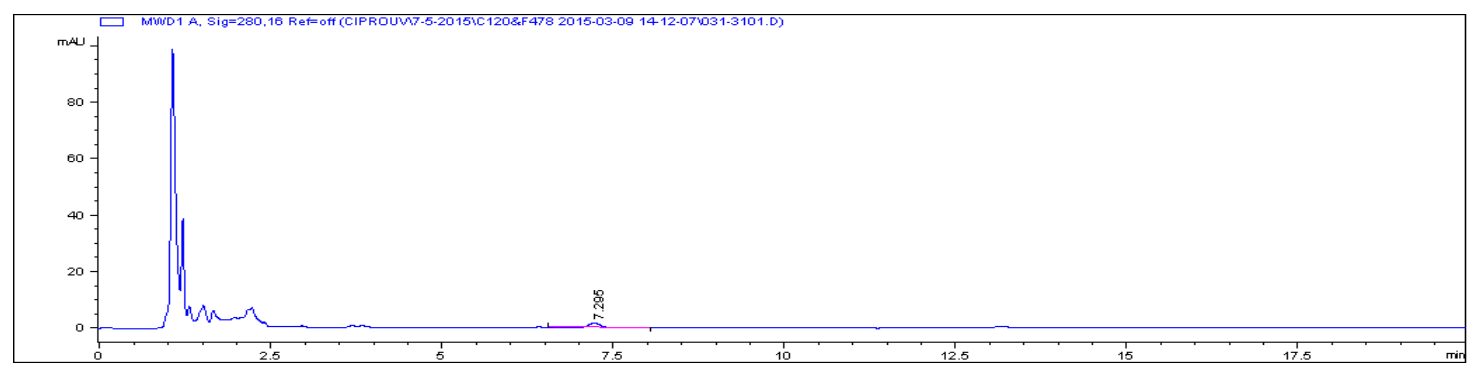

Maximum concentration of ciprofloxacin residues detected in milk collecting centers milk samples (19.25ppb)

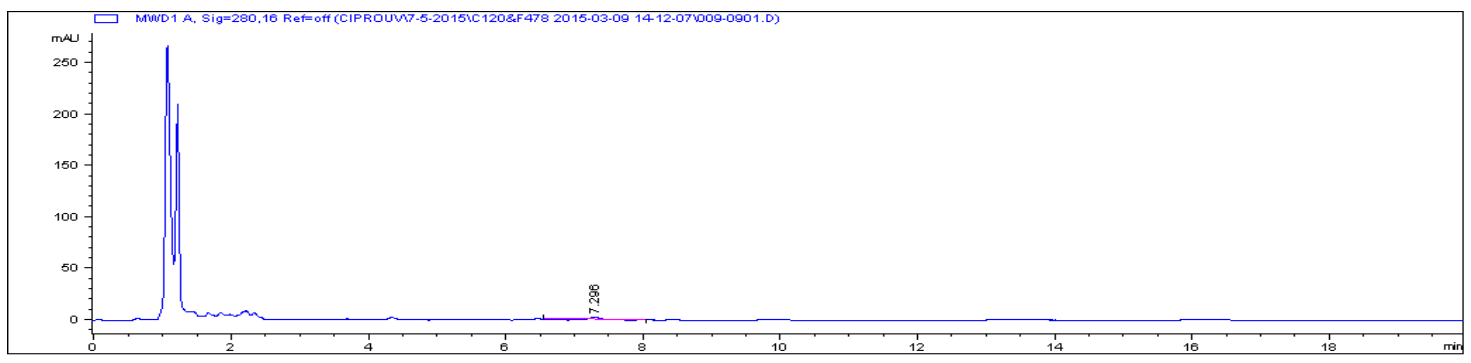

Minimum concentration of ciprofloxacin residues detected in bulk tank milk samples (16.59ppb).

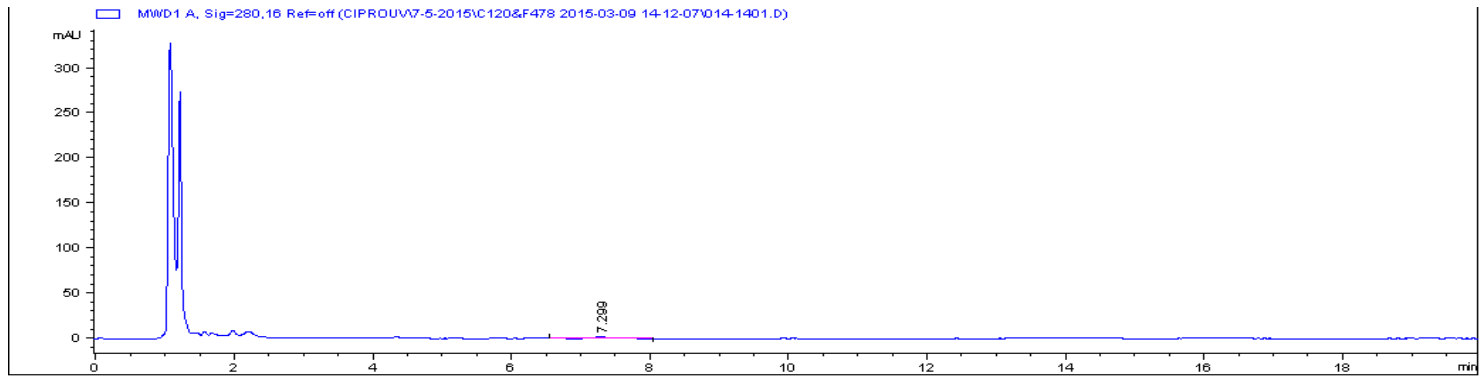

Maximum concentration of ciprofloxacin residues detected in bulk tank milk samples (19.19ppb) 
Table (3) Interpretation of the ciprofloxacin residues in the examined samples with the international standards of permissible ciprofloxacin residues limits

\begin{tabular}{|c|c|c|c|}
\hline Enrofloxacin residues (ppb) & $\begin{array}{c}\mathrm{EU}^{*} \\
(100 \mathrm{ppb})\end{array}$ & $\begin{array}{c}\mathrm{CR}^{* *} \\
(50 \mathrm{ppb})\end{array}$ & $\begin{array}{c}\text { JFCRF*** } \\
(50 \mathrm{ppb})\end{array}$ \\
\hline Market milk (19.47) & Acceptable & Acceptable & Acceptable \\
\hline $\begin{array}{l}\text { Milk collecting centers } \\
(19.25)\end{array}$ & Acceptable & Acceptable & Acceptable \\
\hline Bulk tank milk (19.19) & Acceptable & Acceptable & Acceptable \\
\hline
\end{tabular}

\section{DISCUSSION}

Ciprofloxacin is the cheapest fluoroquinolone and is often prescribed for the treatment of animals (Filip et al., 2014). The administration of fluoroquinolones to foodproducing animals without an adequate withdrawal time may lead to violative concentrations of residues in foods destined for human consumption. These residues represent a risk to public health, including stimulation of bacterial resistance, alterations on intestinal microflora and hypersensitivity reactions (Fabrega et al., 2008). Hence, efficient control of its residues would require screening tests which give rapid results, expected to be simple, cheap, and easy to perform and allow simultaneous analysis of large number of samples (Sanz et al., 2015). Rapid quinolones detection strip test is a rapid (10 min), relatively simple and very easy to use (Cheng et al., 2014).

The percentages of presence of quinolones residues in the examined milk samples were ranged between 14 and $20.6 \%$ (Table 1). Higher results were reported by Navratilova et al. (2011) who recorded that $87.3 \%$ of the raw milk samples were positive for the quinolones residues. While lower results were reported by Aguilera-Luiz et al. (2008) and Nizamlıoglu and Aydın (2012) whom found that none of the examined milk samples were positive for quinolone residues. Presence of quinolones residues in milk may be attributed to their entrance to the food chain either by illegal use or by non-compliance of producers to existing animal-treatment protocols (withdrawal times) prior to milking (Sats et al., 2014). In contrary, absence of quinolones residues in UHT milk may be due to selection and regular monitoring of milk for presence of antimicrobial residues before processing. Factories of dairy products usually use Hazard Analysis and Critical Control Point (HACCP) system in their production plan. The HACCP system approached to minimize economic losses and food poisoning outbreaks from all steps in the dairy production process (Peristeropoulou et al., 2015). Heat treatment may have minor role in reduction of quinolones residues in milk (Roca et al., 2010). The lack of volatility and thermal instability of many antibiotics makes HPLC the best of choice for analysis and confirmation of veterinary drug residues (Kennedy et al., 1998).

Reviewing the data in Table (2) Showed that the percentages of presence of ciprofloxacin in the examined milk samples were ranged from 8 to $11.8 \%$ with a mean values ranged from 17.37 to $18.02 \mathrm{ppb}$. Nearly similar percentages of ciprofloxacin residues were reported by Chowdhury et al. (2015) whom detected ciprofloxacin residues in commercial milk samples $(17 \%$ raw and $16 \%$ boiled) but with lower percentages ( $8 \%$ raw and $5 \%$ boiled) in local cow milk. The higher percentages were reported by Navratilova et al. (2011) whom detected ciprofloxacin residues in $87.3 \%$ of the examined milk samples. Ciprofloxacin is often prescribed for the 
animal treatment so its presence as a residue in milk is predicted; also the frequency of occurrence of ciprofloxacin in the present study may be due to the fact that ciprofloxacin is a marker residue for enrofloxacin. Enrofloxacin is approved for use as a veterinary drug and when it is metabolized, its pharmacologically active metabolite; ciprofloxacin; is produced. Hence detection of ciprofloxacin during market screening often reflects the use of both enrofloxacin and ciprofloxacin (Botsoglou and Fletouris 2001; Navrátilová et al., 2011).

In the current study, it is pointed out that none of positive milk sample has ciprofloxacin residues concentration exceeded maximum residual limits (MRLs) set by different international standards like EU (European Commission 2010)., China regulations (Rong-jie et al., 2014) and Japan Food Chemical Research Foundation (Change et al., 2010) (Table 3). The same results were set by Navratilova et al. (2011). This may be attributed to compliance of producers to existing animal-treatment protocols (withdrawal times) prior to milking (Sats et al., 2014), also high dilution of ciprofloxacin residue in milk by ciprofloxacin residue free milk may reduce ciprofloxacin residues in the examined milk samples. Findings in this study revealed that ciprofloxacin residues were present at low concentrations which can strongly suppress the facultative anaerobic human intestinal microflora (Cerniglia and Kotarski, 1999), and it also leads to the development of resistant bacteria.

The most important side effects of fluoroquinolone include renal impairment, ocular disorders, and damage to articular cartilage. Articular cartilage damage is irreversible and for that reason the preparations containing fluoroquinolone must not be administered to adolescents and pregnant women. Other adverse effects include gastrointestinal disturbances, headache, insomnia, tension, skin allergy manifestations and drug resistance
(Flomenbaum et al., 2006). Several reports have shown that the presence of fluoroquinolone residues in human food obtained from animal sources has reduced the effectiveness of these agents in human medicine. In addition, direct toxicity and other side effects of fluoroquinolone are well established in animals and humans (Gruchalla and Pirmohamed, 2006; Khadra et al., 2012). Therefore, the reduction of antibiotic usage constitutes a challenge for the human health worldwide. Thus it is important to monitor regularly residues of fluoroquinolones in consumed food specially milk.

\section{REFERENCES}

Adekunbi, B.O., Andrew, B.O. 2015. Fluoroquinolone residues in raw meat from open markets in Ibadan, Southwest, Nigeria. J. of Health, Animal sci., and Food safety, (2): 3240.

Aguilera-Luiz, M.M., Vidal, J.L., Gonz, R.R., Frenich, A.G. 2008. Multiresidue determination of veterinary drugs in milk by ultra-high-pressure liquid chromatography-tandem mass spectrometry. J. of Chromatography A, (1205): $10-16$.

Bilandžić, N., Kolanović, B.S., Varenina, I., Jurković, Z. 2011. Concentrations of veterinary drug residues in milk from individual farms in Croatia. J. Mljekarstvo, 61 (3): 260 - 267.

Botsoglou, N.A., Fletouris, D.J. 2001. Drug residues in foods: Pharmacology, Food safety, and Analysis. New York: Marcel Dekker.

Cerniglia, C.E., Kotarski, S. 1999. Evaluation of veterinary drug residues in food for their potential to affect human intestinal microflora. Regulatory Toxicology and Pharmacology. 29, 238-261.

Change, C., Wang, W., Entasin, C. 2010. Simultaneous determination of 18 Quinolone Residues in Marine and Livestock Products by Liquid Chromatography/ Tandem Mass 
Spectrometry. J. Food and Drug Analysis 18(2): 87-97.

Cheng, N., Zhu, L., Xu, Y., Xu, W. 2014. A Review of Test Strips in Rapid Detection of Food Safety. Austin J. Nutri Food Sci., 2 (7): 1038.

Chowdhury, S., Hassan, M.M., Alam, M., Sattar, S., Bari, S., Saifuddin, A.K., Hoque, A. 2015. Antibiotic residues in milk and eggs of commercial and local farms at Chittagong, Bangladesh Veterinary World, EISSN: 2231-0916 Available at www.veterinaryworld.org/Vol.8.

European Commission. 2010. Commission Regulation (EU) No. 37/2010 of 22 312 December 2009: on pharmacologically active substances and their classification regarding maximum residue limits in foodstuffs of animal origin. 313 Official Journal 314 of the European Union, L15, 172.

Fabrega, A., Sa'nchez-Ce 'spedes, J., Soto, S., Vila, J. 2008. Quinolone resistance in the food chain. International Journal of Antimicrobial Agents, 31, 307-315.

Filip, C., Ceana, D., Vescan, A.T. 2014. HPLC method with fluorescence detection for the identification and quantitative determination of Fluoroquinolones in milk. J. Actamedica transil vanica 2 (1): 151 153.

Flomenbaum, N.E., Goldfrank, L.R., Hoffman, R.S., Howland, M.A., Lewin, N.A., Nelson, L.S. 2006. Goldfranks Toxicologic Emergencies. 8th Ed. McGraw-Hill, New York.

Gruchalla, R.S., Pirmohamed, M. 2006. Antibiotic allergy. The New England J. of Med. 354, 601-609.

Juan-García, A., Font, G., Picó, Y. 2006. Determination of quinolone residues in chicken and fish by capillary electrophoresis-mass spectrometry. Electrophoresis. 27, 2240-2249.

Kennedy, D.G., McCracken, R.J., Cannavan, A., Hewitt, S.A. 1998. Use of liquid chromatography-mass spectrometry in the analysis of residues of antibiotics in meat and milk. Journal of Chromatography, A 1067: 93 - 100.

Khadra, A., Pinelli, E., Lacroix, M.Z., Bousquet-Melou, A., Hamdi, H., Merlina, G., Guiresse, M., Hafidi, M. 2012. Assessment of the genotoxicity of quinolone and fluoroquinolones contaminated soil with the Viciafaba micronucleus test. J. of Ecotoxicol., and Environ., Saf., 76, 187-192.

Nadezhda, A., Nataliya, I., Anatoly, V., Sergei, A., Ilya, A., Yuanming, S., Boris, B. 2014. Rapid immunochromatographic assay for ofloxacin in animal original foodstuffs using native antisera labeled by colloidal gold. Talanta. 119 (125-132).

Navratilova, P., Borkovcova, I., Vyhnalkova, J., Vorlova, L. 2011. Fluoroquinolone residues in raw cow's milk. Czech J. Food Sci, (29): 641- 646.

Nizamlığlu, F.O., Aydın, H. 2012. Quinolone antibiotic residues in raw milk and chicken liver in Konya Eurasian. J. Vet Sci 28, 3, 154-158.

Peristeropoulou, M., Fragkaki, A.G., Printzos, N., Laina, I. 2015. Implementation of the Hazard Analysis Critical Control Point (HACCP) System to a Dairy Industry: Evaluation of Benefits and Barriers. J. Food Nutri Diete 1(1): 102.

Roca, M., Castillo, M., Marti, P., Althaus, R.L., Molina, M.P. 2010. the effect milk processing temperatures on the presence of antimicrobial residues. J. Agric Food Chem. 58(9):5427-31.

Rong-jie, F.u., Sun, J.L, Yun-qing, L.I. 2014. Agilent Technologies Shanghai Co. Ltd Analyze Quinolone Residues in Milk with an Agilent Poroshell 120, $4 \mu \mathrm{m} \quad$ Column. www. agilent.com/chem.

Sanz, D., Razquin, P., Condón, S., Juan, T., Herraiz, B., Mata, L. 2015. Incidence 
of Antimicrobial Residues in Meat Using a Broad Spectrum Screening Strategy. European J. of Nut., \& Food Saf., 5(3): 156-165.

Sats, A., Mootse, H., Lepasalu, L., Poikalainen, V. 2014. Use of Delvotest $\mathrm{T}$ for Quantitative Estimation of $\beta$-lactam Antibiotic Residues in Waste Milk and for Evaluation of Thermal Treatment
Efficiency - a Methodical Pilot Study Agronomy Research 12 (3): 807-812. Verdon, E., Couëdor, P., Sanders, P., AfssaLermvd. 2004. Validation of a multiquinolones, multi-matrix, multispecies method for the determination of quinolones residues by HPLC with fluorescence detectin. French Agency for Food Safety, BP 90203, 35302 Fougères, France. 